\title{
R. CASTANET \\ Sémantique formelle des opérateurs d'un langage de listes
}

Revue française d'automatique, informatique, recherche opérationnelle. Informatique théorique, tome 8, $\mathrm{n}^{\circ} \mathrm{R} 3$ (1974), p. 19-36.

$<$ http://www.numdam.org/item?id=ITA_1974_8_3_19_0>

(c) AFCET, 1974, tous droits réservés.

L'accès aux archives de la revue « Revue française d'automatique, informatique, recherche opérationnelle. Informatique théorique » implique l'accord avec les conditions générales d'utilisation (http://www.numdam.org/legal. php). Toute utilisation commerciale ou impression systématique est constitutive d'une infraction pénale. Toute copie ou impression de ce fichier doit contenir la présente mention de copyright.

\section{Numdam}

Article numérisé dans le cadre du programme

Numérisation de documents anciens mathématiques

http://www.numdam.org/ 
R.A.I.R.O.

(8 année, R-3, 1974, p. 19 à 36)

\title{
SEMANTIQUE FORMELLE DES OPERATEURS D'UN LANGAGE DE LISTES
}

\author{
par R. Castanet $\left({ }^{1}\right)$ \\ Communiqué par E. ENGELER
}

\begin{abstract}
Résumé. - Le Lambda K Calculus de Church est un système formel susceptible de servir de langage d'expression de la sémantique d'opérateurs de manipulation de listes et de parcours de ces listes.

On définira les primitives de ces deux types d'opérateurs : extraction, affectation de parties d'éléments composant une liste et on exprimera la sémantique de ces primitives dans le système formel choisi. A partir de là, les opérateurs de parcours de liste et de manipulation (ajout et retrait d'un élément) pourront être de même exprimés.
\end{abstract}

\section{INTRODUCTION}

Formaliser la sémantique d'un opérateur ou d'un objet quelconque d'un langage consiste à définir un système formel dans lequel il est possible d'exprimer cet opérateur ou cet élément. Mais donner une expression dans un certain système formel consiste finalement à paraphraser dans un système dont la sémantique des opérateurs et des objets élémentaires est connue.

La formalisation de la sémantique de plusieurs langages a été réalisée par le calcul des prédicats (Paduceva [5]), par des sous-ensembles de langages (APL pour APL, Lathwell [7], LISP pour LISP, Mac Carthy [9]). Ces études restent attachées à des langages particuliers, sans qu'il soit possible de généraliser ces méthodes à un langage quelconque.

D'après les travaux de Curry [3], L. Nolin [4] et B. Robinet [6], la logique combinatoire représente un domaine où il est possible de définir des systèmes formels adaptés à l'expression de sémantique.

(1) U.E.R. Mathématique et Informatique, Université de Bordeaux I à Talence.

Revue Française d'Automatique, Informatique et Recherche Opérationnelle $\mathrm{n}^{\circ}$ déc. 1974, R-3. 
Ce type de systèmes ont une syntaxe et une sémantique propres très restreintes; ainsi la paraphrase conduit à de très longues expressions pour la sémantique d'un élément d'un langage quelconque.

Curry introduit un combinateur $F$ (fonctionality) spécialisé pour l'expression de la fonction propre d'un opérateur, c'est-à-dire de sa sémantique. B. Robinet a appliqué cette théorie pour les opérateurs du langage APL agissant sur les tableaux.

Cette formalisation nécessite l'introduction d'un combinateur spécifique, alors que le système formel choisi pour cette étude, le Lambda-K-Calculus de Church, n'est absolument pas lié à l'idée de sémantique. Ce système formel permet de proposer une paraphrase dont il est possible de s'inspirer dans une réalisation de micro-programmes pour une machine qui serait conçue pour le traitement de listes. On trouvera en annexe la définition du Lambda-KCalculus. Ce système formel permet d'exprimer tous les opérateurs du langage étudié.

Il est évident que tout système formel ne peut pas toujours exprimer les éléments et les opérateurs d'un langage; dans ce cas, ce système formel serait inadéquat pour exprimer la sémantique du langage total.

Par exemple, les systèmes combinatoires, et, en particulier, le Lambda-KCalculus ne peuvent exprimer l'opération de rupture de séquence $(G \emptyset T \emptyset)$. Par contre, ils sont adaptés à l'expression des traitements conditionnels (IF... THEN... ELSE, WHILE... DO), ainsi qu'au traitement de blocs.

Le langage de listes qui sert de base à cette formalisation est un langage spécialisé dans la manipulation de listes composant un arbre syntaxique associé à une phrase d'un langage naturel. Mais la structure proposée est suffisamment générale pour pouvoir s'adapter à toute autre structure de liste comme celle de LISP [9], SLIP [8], L 6 [10]. On s'intéressera à des opérateurs du langage de base identiques à ceux que l'on peut trouver aussi, explicitement ou implicitement dans tous les langages de listes.

On définira la sémantique des opérateurs du langage par étapes :

- formalisation des éléments simples du langage (paragraphes I, II, III);

- formalisation des primitives des opérateurs du langage (paragraphe IV);

- formalisation des opérateurs eux-mêmes, d'une part de parcours des listes, d'autre part de modification des listes (ajout ou retrait d'un élément) (paragraphe V).

\section{DEFINITION D'UNE LISTE}

Soit $\mathcal{L}$ une liste définie comme un doublet $(S, H)$ tel que $S$ représente l'état statique de la liste $\mathcal{L}$ et $M$ l'ensemble des modificateurs de $S$. 
A la liste $\mathfrak{L}$ est associé un caractère essentiellement dynamique par opposition au caractère statique de $S$.

$S$ est composé d'un ensemble $E$ d'éléments $\left\{e_{i}\right\}, i=[0, n]$ et d'un ensemble $A$ d'accès sur $E$.

Ces accès sont de la forme successeur $s$ ou prédécesseur $p$. Ils font intervenir des informations contenues dans les éléments $\left\{e_{i}\right\}$ de la liste.

L'ensemble $M$ des modificateurs de $S$ est tel que $M=M_{A} \cup M_{R}$ où $M_{A}$ est l'ensemble des modificateurs de type "ajout" et $M_{R}$ l'ensemble des modificateurs de type " retrait".

Pour les ensembles $M$ et $A$ on définit les primitives suivantes :

$a$ : opérateur d'adressage

$c$ : opérateur de contenu

$\rho$ : opérateur de positionnement

$\leftarrow$ : opérateur d'affectation.

Ces primitives ont pu être précisées à la suite de la démarche formalisatrice dans le langage étudié.

On définit $\mathcal{C}$ comme l'ensemble des valeurs pouvant être contenues dans un élément $e_{i} \in E$, et $\mathcal{A}$ comme l'ensemble des valeurs de $\mathcal{C}$ pouvant être des adresses.

Ainsi :

$a$ : est un opérateur de $\mathcal{A}$ dans $E$

$c$ : est un opérateur de $E$ dans $\mathcal{C}$

$\rho$ : est un opérateur de $E$ dans $E$.

\section{SEMANTIQUE D'UN ELEMENT $e_{i} \in E$}

Dans toute formalisation d'un langage, il est indispensable de considérer, d'une part, les primitives des opérateurs de ce langage, et, d'autre part, les éléments primitifs sur lesquels agissent les opérateurs.

Un élément $e_{i}$ avait été présenté dans un souci de clarté comme le plus petit élément accessible de la liste.

En réalité dans toute liste, ces éléments nommés cellules de liste, doivent être décomposés.

On propose une décomposition très générale qui pourrait être simplifiée pour certains langages de listes comme LISP, c'est-à-dire que certains niveaux de la décomposition ne seront pas présents.

nº́cembre 1974, R-3. 
Définition 1. Un élément $e_{i} \in E$ est un $n$-uple ayant une structure hiérarchisée telle que :

$$
\begin{aligned}
& e_{i}=\{\operatorname{mot}\}^{m}: \text { ensemble de } m \text { mots concaténés } \\
& \text { mot }=\text { reliquat } \cup \text { partie information } \\
& \text { partie information }(\mathrm{p} . \mathrm{i} .)=\{\text { caractère }\}^{p} \\
& \text { reliquat }=\{\text { composante élémentaire (c.e.) }\}^{r} \\
& \text { caractère }=\{\text { composante élémentaire (c.e.) }\}^{c}
\end{aligned}
$$

Les éléments primitifs de $\mathfrak{L}$ sont les éléments « mot », " reliquat », " partie information », « caractère », " composante élémentaire ».

\section{EXPRESSION D'UN ELEMENT $e_{i}$ DANS LE SYSTËME FORMEL}

On représentera le $n$-uple $e_{i}$ par la Lambda $K$ expression :

$$
\lambda x\left(x x_{1} x_{2} \ldots x_{n-1} x_{n}\right)
$$

c'est un vecteur qui représente la structure physique d'un $e_{i}$, alors que la structure logique d'un $e_{i}$ est une arborescence avec les niveaux successifs mot, p.i., caractère, c.e.

\section{EXPRESSION DES PRIMITIVES DES OPERATEURS DU LANGAGE DE LISTE}

Définition 2. Soit $M, A, F, B, C \in V$ ensemble des vecteurs et $E_{x}$ ensemble des éléments du vecteur $x$ tel que $E_{M}, E_{A}, E_{B}, E_{C} \in E_{e_{i}} . M, A, F, B, C$ sont des $i$-uples $1 \leqslant i<n$.

Soit $J$ l'ensemble des entiers positifs ou nuls : $N, D, E, P \in J$ et sont inférieurs ou égaux à $n$.

On définit des opérateurs contenu ou opérateurs d'extraction :

$\mathcal{H}$ opérateur d'extraction d'un mot

$\mathcal{A}$ opérateur d'extraction d'une p.i.

$\mathcal{R}$ opérateur d'extraction d'un reliquat

$\beta$ opérateur d'extraction d'une c.e.

C opérateur d'extraction d'un caractère.

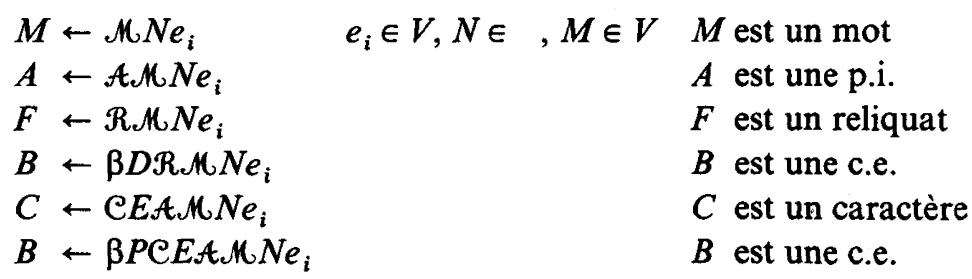


Proposition 1. La sémantique de l'opérateur contenu sur les composantes d'un élément d'une liste peut être représenté par un combinateur $\mathcal{G}$ 2-paramétré exprimable dans le système formel du Lambda K-Calculus par :

$$
\mathcal{G}_{x, y}=\lambda u_{1} \lambda u_{2}\left(\lambda v \left(v \Re\left(x\left(P u_{1}\right)\right) W\left(\left(K u_{2}\right) \varepsilon\right)(P y) W((K I(J) K))\right.\right.
$$

$x, y$ sont les paramètres du combinateur $\mathbf{G}$.

Les combinateurs $X, P, W, K, \mathcal{E}, I, J$ sont $\lambda$-K exprimables

$X=\lambda m_{1} \lambda m_{2} \lambda a\left(m_{1}\left(m_{2} a\right)\right.$ multiplication dans l'ensemble des entiers $\mathfrak{J}$

$P=$ prédécesseur dans $\mathfrak{J}$

$W=\lambda r_{1} r_{2}\left(r_{1} r_{2} r_{2}\right)$

$K=\lambda x y(x)$

$\mathcal{E}=\lambda x y(y)$

$I=\lambda x(x)$ identité

$J=\lambda x(\lambda y x y)$.

On a les équivalences suivantes d'après la définition II :

$$
\begin{aligned}
& \mathcal{M} \equiv \mathcal{S}_{n, n} \\
& \mathcal{C} \equiv \mathfrak{G}_{a, a} \\
& \beta \equiv \mathcal{G}_{1,1} \\
& \mathcal{R} \equiv \mathfrak{S}_{0, P_{r}} \quad \text { (prédécesseur de } r \text { ) } \\
& \mathcal{A} \equiv \mathcal{S}_{r, r P_{n}} \quad(n-r: r \text { fois le prédécesseur de } n) \text {. }
\end{aligned}
$$

Remarque : $n, a, r$ sont des entiers définissant la structure d'un $e_{i}$.

$$
\begin{aligned}
& n: \text { nombre de c.e. d'un mot } \\
& a: \text { nombre de c.e. d'un caractère } \\
& r: \text { nombre de c.e. d'un reliquat. }
\end{aligned}
$$

Ces entiers sont suffisants pour définir la structure d'un $e_{i}$. Il est possible de trouver une autre combinaison de 3 entiers pour définir un $e_{i}(3$ entiers : nombre de niveaux de la structure hiérarchisée d'un $e_{i}$ moins une unité).

Démonstration de la proposition 1 pour $\mathfrak{G}_{n, n}$

Extraction du contenu du Nième mot de la cellule $e_{i}$

$$
\begin{aligned}
\mathfrak{S}_{n, n} N e_{i} & =\lambda u_{1} \lambda u_{2}\left(\lambda v \left(v \mathscr{X}\left(n\left(P u_{1}\right)\right) W\left(\left(K u_{2}\right) \mathcal{E}\right)(P n) W((K I(J) K)) N e_{i}\right.\right. \\
& =\lambda v\left(v \mathscr{X}(n(P N)) W\left(K e_{i}\right) \mathcal{E}\right)(P n) W((K I(J) K)) .
\end{aligned}
$$

On décompose cette expression afin d'en rendre plus clair le calcul :

$$
A=\mathfrak{X}(n(P N)) W\left(K e_{i}\right) \mathcal{E} \quad, \quad B=(P n) W((K I(J) K)) .
$$

Dans ce système formel les entiers sont représentés par l'expression suivante :

$$
\forall n \geqslant 0 \equiv \lambda n_{1} n_{2}\left\{\left(n_{1}\right\}^{n} n_{2}\{)\right\}^{n} \text { si }\left\{\left(n_{1}\right\}^{n}\right.
$$

nº́cembre 1974, R-3. 
représente la concaténation de $n$ fois « $\left(n_{1} \text { " et }\{)\right\}^{n}$ la concaténation de $n$ parenthèses fermantes.

$$
\begin{aligned}
A & =\lambda n_{1} n_{2}\left\{\left(n_{1}\right\}^{n \times(N-1)} n_{2}\{)\right\}^{n \times(N-1)} W\left(\left(K e_{i}\right) \mathcal{E}\right) \\
& =\left\{(W\}^{n(N-1)}\left(K e_{i}\right) \mathcal{E}\{)\right\}^{n \times(N-1)}
\end{aligned}
$$

L'expression de $W$ étant $\lambda r_{1} r_{2}\left(r_{1} r_{2} r_{2}\right)$ on a :W(Ke $e_{i} \mathcal{E}=K e_{i} \varepsilon \mathcal{E}$.

Ainsi $A=K e_{i}\{\mathcal{E}\}^{n \times(\hat{N}-1)+1}=\lambda x y(x) e_{i}\{\delta\}^{n \times(N-1)+1}=e_{i}\{\mathcal{E}\}^{n \times(N-1)}$

L'expression de $e_{i}$ étant $\lambda x\left(x x_{1} \ldots x_{p}\right)$ on a $e_{i} \delta=\delta x_{1} \ldots x_{p}=\lambda y\left(y x_{2} \ldots x_{p}\right)$.

Ainsi on obtient l'élimination de $n \times(N-1)$ premiers éléments de $e_{i}$ par application de $\delta n \times(N-1)$ fois.

Ainsi :

$$
\begin{aligned}
& A=\lambda x\left(x x_{n \times(N-1)+1} \ldots x_{p}\right) \\
& B=(P n) W((K I) J) K
\end{aligned}
$$

Par une suite de transformations analogues :

$$
B=K I\{J\}^{n} K=\{J\}^{n-1} K
$$

$B$ appliqué sur $A$ nous permet de sélectionner les $n$ premiers éléments de $A$ :

$$
\begin{aligned}
A B & =\lambda x\left(x x_{n \times(N-1)+1} \ldots x_{p}\right)\{J\}^{n-1} K=J x_{n \times(N-1)+1} \ldots x_{p}\{J\}^{n-2} K \\
& =x_{n \times(N+1)+1} \ldots \lambda y\left(x_{n \times N-1} y x_{n+N} \ldots x_{p}\right) K=x_{n \times(N-1)+1} \ldots x_{n \times N}
\end{aligned}
$$

Ainsi $\mathcal{G}_{n, n} N e_{i}=\lambda v\left(v x_{n \times(N-1)+1} \ldots x_{n \times N}\right)$.

Définition 3. Soient $\mathfrak{T}_{m}, \mathfrak{T}_{c}, \mathfrak{T}_{B}, \mathfrak{T}_{i}$ les opérateurs de positionnement sur respectivement : un mot, un caractère, une composante élémentaire et une partie information, tels que :

$$
\mathfrak{T}_{m} N e_{i} \rightarrow V_{1}^{*} \quad, \quad \mathfrak{T}_{i} V_{1}^{*} \rightarrow V_{2}^{*} \quad, \quad \mathfrak{T}_{c} C \dot{V}_{2}^{*} \rightarrow V_{3}^{*} \quad, \quad \mathfrak{T}_{B} B V_{3}^{*} \rightarrow V_{4}^{*}
$$

On a : $N, C, B \in \mathfrak{J}$ ensemble des entiers naturels. $V_{i}^{*} \notin V$ ensemble des vecteurs si $N, C, B \neq 0$; on a $V_{i}^{*} \in V^{*}$ ensemble de vecteurs pointés (un vecteur pointé possède des composantes comme un vecteur, mais l'une d'elles est repérée ou pointée. Exemple : $x_{1} x_{2} \ldots x_{k} \lambda x\left(x x_{k+1} \ldots x_{n}\right)$ est un vecteur pointé avec la composante $x_{k+1}$ pontée).

Proposition 3. La sémantique de l'opérateur de positionnement sur un vecteur ou un vecteur pointé peut être représentée par un combinateur $\pi$ 1-paramétré exprimable dans le système formel du Lambda $K$-calculus :

$$
\pi_{x}=\lambda u_{1} \lambda u_{2}\left(x\left(x\left(P u_{1}\right)\right) W\left(\left(K u_{2}\right) J\right)\right) .
$$

On a les équivalences :

$$
\pi_{n} \equiv \mathfrak{T}_{m} \quad, \quad \pi_{r} \equiv \mathfrak{T}_{i} \quad, \quad \pi_{e} \equiv \mathfrak{T}_{c} \quad, \quad \pi_{1} \equiv \mathfrak{T}_{B}
$$

La démonstration de cette proposition ne pose aucune difficulté. 
Définition 4. Soient $\mathcal{R}_{m}, \mathcal{R}_{r}, \mathcal{R}_{p}, \mathcal{R}_{c}, \mathcal{R}_{B}$ les opérateurs d'affectation, respectivement d'un mot, d'un reliquat, d'une p.i., d'un caractère, d'une c.e. Ces opérateurs agissent sur le résultat d'un positionnement. Ils effectuent une élimination (remise à zéro) suivie d'une insertion.

Ces opérateurs envoient l'ensemble des vecteurs pointés $V^{*}$ et des vecteurs $V$ sur l'ensemble des vecteurs $V$.

Proposition 3. La sémantique de l'opérateur d'affectation sur un vecteur pointé peut être représenté par un combinateur $A$ 1-paramétré exprimable dans le système formel du Lambda $K$ calculus.

$$
\begin{gathered}
\mathcal{A}_{x}=\lambda u_{1} \lambda u_{2}\left(\lambda v\left(v x W\left(\left(K u_{2}\right) \mathcal{E}\right)(\operatorname{Pr}) W\left(\left(K u_{1}\right)\left(J_{1} J\right)\right)\left(K_{1} J\right) I\right)\right) \\
J_{1}=\lambda x y z((x y) z) \quad \text { extension de } J \text { à } 3 \text { variables } \\
K_{1}=\lambda x y(\lambda z(x y)) \quad \text { extension de } K \text { à } 3 \text { variables. }
\end{gathered}
$$

On a les équivalences :

$$
\mathcal{R}_{m} \equiv \mathcal{A}_{n} \quad, \quad \mathcal{R}_{r} \equiv \mathcal{A}_{r} \quad, \quad \mathcal{R}_{p} \equiv \mathcal{A}_{n-r} \quad, \quad \mathcal{R}_{e} \equiv \mathcal{A}_{e} \quad, \quad \mathcal{R}_{B} \equiv \mathcal{A}_{1} .
$$

Démonstration pour $\mathfrak{A}_{n}$ par exemple :

$$
\begin{gathered}
\mathcal{A}_{n} V_{i}^{*} V_{j} \rightarrow V_{i} \quad V_{i}, V_{j} \in V \text { vecteurs } \\
\quad V_{i}^{*} \in V^{*} \text { vecteurs pointés. } \\
\begin{aligned}
\mathcal{A}_{n} V_{i}^{*} V_{j} & =\lambda v\left(v\left(n W\left(\left(K V_{i}^{*}\right) \mathcal{E}\right)(P n) W\left(\left(K V_{j}^{*}\right)\left(J_{1} J\right)\right)\left(K_{1} J\right) I\right)\right) \\
& =\lambda v\left(v V_{i}^{*}\{\mathcal{E}\}^{n} V_{j}\left\{\left(J_{1} J\right)\right\}^{n-1}\left(K_{1} J\right) I\right) .
\end{aligned}
\end{gathered}
$$

$V_{i}^{*}$ est de la forme : $x_{1} x_{2} \ldots x_{k} \lambda y\left(x_{k+1} y \ldots x_{p}\right)$.

$V_{i}^{*}\{\delta\}^{n}$ provoque l'élimination des $n$ premières composantes de $V_{i}^{*}$ à partir de l'élément pointé.

$V_{j}$ est de la forme : $\lambda y\left(y x_{1}^{\prime} \ldots x_{n}^{\prime}\right)$

$V_{j}\left\{\left(J_{1} J\right)\right\}^{n-1}$ permet d'obtenir la chaîne : $\left(J x_{1}^{\prime}\right)\left(J x_{2}^{\prime}\right) \ldots \lambda z\left(\left(J x_{n-1}^{\prime}\right) z x_{n}^{\prime}\right)$.

On peut ainsi insérer dans le vecteur pointé $V_{i}^{*}$ les composantes de $V_{j}$; on obtient finalement un vecteur $V_{i}$ tel que :

$$
V_{i}=\lambda v\left(v x_{1} \ldots x_{n \times(N-1)-1} x_{1}^{\prime} x_{2}^{\prime} \ldots x_{n}^{\prime} x_{n \times(N)+1} \ldots x_{p}\right)
$$

Définition 5. On peut représenter l'opérateur d'adressage $D$ dans une mémoire à bande par le combinateur d'extraction $\mathfrak{S}_{l, l}$ dans lequel $l$ est le nombre de composantes de cette mémoire.

$\mathfrak{S}_{l, l}$ envoie l'ensemble $\mathfrak{J} \times \mathcal{M}$ sur l'ensemble $E$. (J ensemble des entiers, $\mathcal{H}$ ensemble des mémoires, $E$ ensemble des cellules.) 


\section{EXPRESSION DE LA SEMANTIQUE DES OPERATEURS D'UN LANGAGE DE LISTE}

\section{V.1. Opérateurs $\in A$ (accès sur $E$ )}

Pour définir précisément ces opérateurs, il est indispensable de se donner un type de liste et sa représentation.

On s'intéressera à une liste symétrique du type de celles du LISP [9].

On se limitera à l'étude de quelques opérateurs d'accès. Soit la structure de liste suivante :

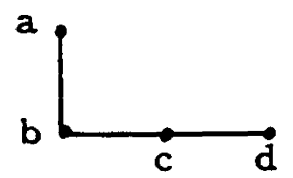

telle que $b, c, d$ sont les trois successeurs directs de $a$.

Sur cette structure on définit, à l'aide des primitives $a, \rho, c, \rightarrow$ (paragraphe I) et du pointeur de niveau $\pi$ les opérateurs suivants :

DESCG (descente à gauche $: a \rightarrow b$ ) $\equiv c \cdot \rho^{1} \cdot a \cdot c \cdot \pi$

DROITE (aller à droite $: b \rightarrow c, c \rightarrow d$ ) $\equiv c \cdot \rho^{2} \cdot a \cdot c \cdot \pi$

GAUCHE (aller à gauche) : $(d \rightarrow c, c \rightarrow b) \equiv c \cdot \rho^{3} \cdot a \cdot c \cdot \pi$

DESCD (descente à droite $: a \rightarrow d) \equiv\left\{c \cdot \rho^{2} \cdot a \cdot c\right\}^{n} \cdot c \cdot \rho^{1} \cdot a \cdot c \cdot \pi$

HAUT (prédécesseur : $d \rightarrow a, c \rightarrow a, b \rightarrow a) \equiv c \cdot \rho^{3} \cdot a \cdot\left\{c \cdot \rho^{3} \cdot a \cdot c\right\}^{p-1} \cdot \pi$

Ces différents opérateurs indiquent qu'un élément de liste $e_{i}$ contient au moins quatre sous-éléments avec le second contenant une information pour DESCG (présence de $\rho^{1}$ ), le troisième une information pour DROITE (présence de $\rho^{2}$ ), le quatrième une information pour GAUCHE ou HAUT (présence de $\rho^{3}$ ).

Sémantiquement, on peut définir deux classes d'opérateurs : mouvement simple : $\quad$ \{DESCG, GAUCHE, DROITE mouvement multiple : \{HAUT, DESCD \}.

Expression des opérateurs de mouvement simple :

Proposition 4. La sémantique des opérateurs de mouvement peut s'exprimer sous la forme d'un couple de combinateurs $\left(M^{1}, M^{2}\right)$ 1-paramétré par $u$ affectant le pointeur de liste $\pi$ tels que :

$$
M_{p_{1}}^{1} \equiv \mathcal{R}_{p} V_{p}^{\prime} \mathfrak{T}_{i} 1 \quad \text { et } \quad M^{2} \equiv \mathcal{R}_{B} i \mathfrak{T}_{B} 4
$$


avec :

$$
\begin{aligned}
& \mid \begin{array}{l}
V_{p}^{\prime} \leftarrow \mathcal{A} i \pi V_{p} \\
V_{p} \leftarrow \mathcal{A} u D \mathcal{A} \pi \\
i \leftarrow \beta 2 \mathfrak{R} V_{p}
\end{array} \\
& \pi \leftarrow\left(M_{u}^{1}, M^{2}\right) \cdot \pi
\end{aligned}
$$

et :

On rappelle que les combinateurs élémentaires présents dans le couple $\left(M_{u}^{1}, M^{2}\right)$ signifient :

$\mathcal{T}_{B}$ : positionnement sur une composante élémentaire

$\mathcal{T}_{i}$ : positionnement sur une partie information

$\mathcal{R}_{B}$ : affectation d'une composante élémentaire

$\mathcal{R}_{p}:$ affectation d'une partie information

$\mathcal{A}$ : contenu d'une partie information

$\mathcal{N}$ : contenu d'un mot

$\beta$ : contenu d'une composante élémentaire

$\mathcal{R}$ : contenu d'un reliquat

$D$ : combinateur d'adressage.

Le combinateur $M_{u}^{1}$ permet d'extraire la partie information du uième mot de la cellule pointée par $\pi$ et d'affecter la p.i. de $\pi$ avec cette information. Cette opération ne s'effectue que si la $2 e$ c.e. du $u$ ième reliquat de $e_{i}$ contient $\lambda x(x 0)$; sinon cette c.e. contient le combinateur $\lambda x(x K)$ et $\pi$ reste inchangé (il n'est pas possible d'effectuer le mouvement simple demandé : par exemple pour DESCG, l'élément pointé par $\pi$ n'a pas de successeur).

Le combinateur $M^{2}$ permet de stocker le résultat de l'opération exprimé sous forme : $\{\lambda x(x 0), \lambda x(x K)\}$ dans la $4 e$ c.e. du reliquat de $\pi$.

Démonstration de la proposition 4 pour l'opération DESCG

Dans ce cas : $u=2$.

$$
\begin{array}{ll}
V_{p} \leftarrow \mathcal{H} 2 D \mathcal{A} \pi & , \quad V_{p}: 2^{\mathrm{e}} \text { mot de la cellule pointée par } \pi \\
i \leftarrow \beta 2 \mathcal{R} V_{p} & , \quad i: 2^{\mathrm{e}} \text { c.e. du reliquat de } V_{p}
\end{array}
$$

1) $\mathrm{Si} \quad i \equiv \lambda x(x 0)$ on a : $V_{p}^{\prime} \leftarrow \mathcal{A} i \pi V_{p}$

$$
\text { ou } V_{p}^{\prime} \leftarrow \mathcal{A} 0 \pi V_{p} \text { ou : } V_{p}^{\prime} \leftarrow \mathcal{A} V_{p}, V_{p}^{\prime}: \text { p.i. de } V_{p}
$$

$\mathfrak{T}_{i} 1 \pi$ permet le positionnement sur la p.i. de $\pi$ et $\mathcal{R}_{p} V_{p}^{\prime} \mathfrak{T}_{i} 1 \pi$ affecte p.i. de $\pi$ avec $V_{p}^{\prime}$.

2) Si $i \equiv \lambda x(x K)$ on a $: V_{p}^{\prime} \leftarrow \mathcal{A} K \pi V_{p}$ c'est-à-dire $V_{p}^{\prime} \leftarrow \mathcal{A} \pi$. Cela revient à affecter la p.i. de $\pi$ avec p.i. de $\pi$, donc à ne pas modifier $\pi$.

Le combinateur $M^{2}$ du couple est tel que $: \pi \leftarrow M^{2} \pi, \pi \leftarrow \mathfrak{R} i \mathcal{S} 4 \pi$.

$$
\left\{\begin{array}{l}
\mathfrak{T}_{B} 4 \pi \text { positionnement sur la } 4^{\text {e }} \text { c.e. du reliquat de } \pi \\
\mathcal{R}_{B} i \mathfrak{T}_{B} 4 \pi \text { remplacement par } i \text { de cette c.e. }
\end{array}\right.
$$

nº décembre 1974, R-3. 
Expression des opérateurs de mouvement multiple

Pour ce type d'opérations, il faut introduire une récursivité afin de répéter autant de fois qu'il est nécessaire l'opération de mouvement simple associée.

Par exemple, dans la structure suivante :

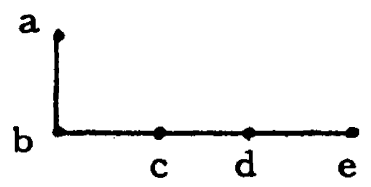

l'opération HAUT à partir de $d$ est équivalente à deux opérations GAUCHE, suivies d'une montée directe.

De même, pour cet exemple, l'opération DESCD (descente à droite) équivaut à DESCG suivie de trois opérations DROITE.

La répétition des mouvements est obtenue par la présence d'un combinateur dans la cellule pointée à chaque mouvement élémentaire suivant le même procédé que pour l'adressage indirect dans la technologie des ordinateurs (cet adressage se présente généralement sous la forme d'un astérisque. Dans l'exemple pris, on aura $\epsilon \cdot b$ (contenu de $b$ ) $=* a, \epsilon \cdot c=* b, \epsilon \cdot d=* c$, $\epsilon \cdot e=* d$. Ainsi, à partir de $e$, on accèdera à l'élément $a$ par 3 indirections).

\section{Expression de l'opérateur HAUT}

Soit $p$ l'opérateur prédécesseur et $S^{i}$ l'opérateur donnant le ième successeur. On suppose que l'on a c.e. ${ }^{3}$ (reliquat $\left.(\operatorname{mot} 4)\right) e_{i}$ égale à :

avec :

$$
\begin{array}{lll}
\lambda x(x 0) & \text { si } & p\left(e_{i}\right)=\varnothing \\
\lambda x(x K) & \text { si } & e_{i}^{*}\left(e_{i} \text { courant }\right)=S^{1}\left(e_{i}\right) \\
\lambda x(x R) & \text { si } & e_{i}^{*}=S^{i}\left(e_{i}\right) \quad i>1
\end{array}
$$

D'autre part, comme pour les autres opérateurs, on supposera que c.e $e^{5}$. (reliquat $(\operatorname{mot} 2)) e_{i}^{*}$ est égale à :

$$
\begin{array}{lll}
\lambda x(x 0) & \text { si } & p\left(e_{i}^{*}\right) \neq \varnothing \\
\lambda x(x K) & \text { si } & p\left(e_{i}\right)=\varnothing \quad \text { (tête de liste) } .
\end{array}
$$

Proposition 5. La sémantique de l'opérateur HAUT s'exprime par un couple de combinateur $\left(H^{1}, H^{2}\right)$ sur $\pi$ tels que :

$$
\begin{aligned}
H_{1}: \pi \leftarrow \mathcal{R}_{p} \mathcal{A}_{i} I V_{4} \pi \mathcal{T}_{i} 1 \pi & V_{4} \leftarrow \mathcal{H} 4 D \mathcal{A} \pi \\
H_{2}: \pi \leftarrow \mathcal{R}_{B} \beta 5 \mathcal{R} V_{4} \mathfrak{T}_{B} 4 \pi & i \leftarrow \beta 5 \mathcal{R} V_{4}
\end{aligned}
$$




\section{Démonstration :}

- si $i=\lambda x(x 0) \quad \mathcal{R}_{p} \mathcal{A}_{i} I V_{4} \pi \mathfrak{T}_{i} 1 \pi \Rightarrow \mathcal{R}_{p} \mathcal{A} 0 V_{4} \pi \mathfrak{T}_{r} 1 \pi$

$$
\Rightarrow \mathcal{R}_{p} \mathfrak{A} \pi \mathfrak{S}_{r} 1 \pi: \pi \text { inchangé. }
$$

- si $i=\lambda x(x K) \quad \mathcal{R}_{p} A \lambda x x(x K) I V_{4} \pi \mathcal{S}_{i} 1 \pi$

$$
\begin{aligned}
& \Rightarrow \mathcal{R}_{p} A K V_{4} \pi \mathscr{S}_{r} 1 \pi \\
& \Rightarrow \mathcal{R}_{p} A V_{4} \mathfrak{S}_{r} 1 \pi: \text { p.i. }(V) \rightarrow \text { p.i. }(\pi)
\end{aligned}
$$

- si $i=\lambda x(x R) \quad \mathcal{R}_{p} \mathcal{A} \lambda x(x R) I V_{4} \pi \mathfrak{S}_{i} 1 \pi$

$$
\begin{aligned}
& \Rightarrow \mathcal{R}_{p} A R V_{4} \pi \mathfrak{T}_{i} 1 \pi \\
& \Rightarrow \mathcal{R}_{p} \mathcal{A} \beta 5 \mathcal{R} r \mathcal{M}_{4} D \mathcal{A} V_{4} \pi \mathfrak{T}_{i} 1 \pi \\
& \Rightarrow \mathcal{R}_{p} \mathfrak{A} \beta 5 \mathcal{R} V_{4}^{\prime} \pi \mathfrak{T}_{i} 1 \pi \\
& \Rightarrow \mathcal{R}_{p} \mathcal{A} \underbrace{\beta 5 \Re V_{4}^{\prime}} I V_{4}^{\prime} \pi \mathfrak{T}_{i} 1 \pi
\end{aligned}
$$

La partie en accolade permet d'atteindre une nouvelle composante $i^{\prime}$

$$
\Rightarrow \Re_{p} \mathfrak{A} i^{\prime} I V_{4}^{\prime} \pi \mathfrak{T}_{i} 1 \pi
$$

Cette forme est équivalente à la forme primitive de l'opérateur HAUT $i$ et $V_{4}$ ont été remplacés par $i^{\prime}$ et $V_{4}^{\prime}$.

Pour continuer le processus itératif, il faut examiner la valeur de $i^{\prime}$. La dernière itération se fera lorsqu'on arrivera en début de niveau, c'est-à-dire lorsque $i^{(x)}=\lambda x(x K)$.

Une condition nécessaire pour que l'opérateur HAUT soit applicable quel que soit $e_{i}$ est que $R$ ne contienne aucune variable liée telle que $\pi$ ou une variable dépendant d'un $e_{i}$. La forme présentée satisfait cette condition.

Expression de l'opérateur DESCD (descente à droite)

Soit $s$ l'opérateur donnant les successeurs de $e_{i}$.

$\mathrm{Si} \quad \operatorname{card}\left(s\left(e_{i}\right)\right)=n \quad$ DESCD $\equiv$ DESCG $\{\text { DROITE }\}^{n}$

Soit $S^{i}\left(e_{i}\right)$ le ième successeur de $e_{i}$, on suppose que l'on a pour $S^{1}\left(e_{i}\right)$ et pour $S^{i}\left(e_{i}\right)$ avec $i \neq 1$, c.e $^{3}$. (reliquat $\left.\left(\operatorname{mot}^{4}\right)\right)$ égal à :

- $\lambda x(x 0)$ si $S^{1}\left(e_{i}\right)=\varnothing$ (vide) ou DROITE $\left(e_{i}^{*}\right)=\varnothing\left(e_{i}^{*}\right.$ étant l'élément $e_{i}$ courant dans l'itération).

$-\lambda x\left(x R^{\prime}\right)$ si $S^{1}\left(e_{i}\right) \neq \varnothing \quad$ et $\quad$ DROITE $\left(e_{i}^{*}\right) \neq \varnothing$

$$
R^{\prime} \equiv \beta 3 \mathcal{R} r \mathcal{H} 3 D \mathcal{A} K \text { avec } K^{\prime}=\lambda x y(x) \text {. }
$$

D'autre part, on a :

c. $e^{4}$. (reliquat $\left.\left(\operatorname{mot}^{2}\right)\right) e_{i}$ égale à :

$-\lambda x(x 0)$ si $s\left(e_{i}\right) \neq \varnothing$

$-\lambda x(x K)$ sinon.

$n^{\circ}$ décembre 1974, R-3. 
Ainsi, au premier pas de l'itération, on peut connaître le résultat de l'opérateur, si $s\left(e_{i}\right) \neq \varnothing$, résultat $\Leftrightarrow \lambda x(x 0)$, sinon résultat $\Rightarrow \lambda x(x K)$.

Proposition 6. La sémantique de l'opérateur DESCG s'exprime par un couple de combinateurs sur $\pi\left(D_{1}, D_{2}\right)$ tels que :

$$
\begin{array}{ll|l}
D_{1}: \pi \leftarrow \mathcal{R}_{p} \mathcal{A} i I V_{2} \pi \mathfrak{T}_{i} 1 \pi & \text { avec } & \begin{array}{l}
V_{2} \leftarrow \mathcal{H} 2 D \mathcal{A} \pi \\
\end{array} \\
D_{2}: \pi \leftarrow \mathcal{R} \beta 4 \mathcal{R} V_{4} \mathfrak{T} 4 \pi . &
\end{array}
$$

Démonstration :

Cas $n^{\circ} 1: s\left(e_{i}\right)=0 \Rightarrow i=\lambda x(x 0)$

$$
\begin{aligned}
& \mathcal{R}_{p} \mathfrak{A} \lambda x(x 0) I V_{2} \pi \mathfrak{T}_{i} 1 \pi \\
& \Rightarrow \mathcal{R}_{p} \mathfrak{A} 0 V_{2} \pi \mathcal{T}_{i} 1 \pi \\
& \Rightarrow \mathcal{R}_{p} \mathfrak{A} \pi \mathfrak{T}_{i} 1 \pi: \text { p.i. }(\pi) \rightarrow \text { p.i. }(\pi) \quad, \quad \pi \text { inchangé. }
\end{aligned}
$$

Cas $n^{\circ} 2:\left|s\left(e_{i}\right)\right|=1 i=\lambda x\left(x R^{\prime}\right)$

$$
\begin{aligned}
& \mathcal{R}_{p} \mathcal{A} R^{\prime} V_{2} \pi \mathfrak{f}_{i} 1 \pi \Rightarrow \mathcal{R}_{p} \mathcal{A} \beta 3 \mathcal{R} r \mathcal{H} 3 D A K^{\prime} V_{2} \pi \mathfrak{f}_{i} 1 \pi \\
& \Rightarrow \mathcal{R}_{p} \mathcal{A} \beta 3 \mathcal{R} r \underbrace{\mathcal{H} 3 D \mathcal{A} V_{2}}_{V_{3}^{\prime}} V_{2} \mathfrak{T}_{i} 1 \pi \\
& \Rightarrow \mathcal{R}_{p} \mathcal{A} \underbrace{\beta 3 \mathcal{R} V_{3}^{\prime}}_{i^{\prime}} I V_{3}^{\prime} V_{2} \mathfrak{I}_{i} 1 \pi
\end{aligned}
$$

$$
\begin{gathered}
\operatorname{Card}\left(s\left(e_{i}\right)\right)=1 \quad i^{\prime}=\lambda x(x 0) \\
\mathcal{R}_{p} \mathfrak{A} R^{\prime} V_{2} \pi \mathscr{T}_{i} 1 \pi \Rightarrow \mathcal{R}_{p} \mathfrak{A} V_{3}^{\prime} V_{2} \mathfrak{S}_{i} 1 \pi \Rightarrow \mathcal{R}_{p} \mathfrak{A} V_{2} \mathfrak{S}_{i} 1 \pi
\end{gathered}
$$

Ainsi $s\left(e_{i}\right) \rightarrow$ p.i. $(\pi)$.

Cas $n^{\circ} 3:$ Card $\left(s\left(e_{i}\right)\right)>1$

$$
i^{\prime}=i=\lambda x\left(x R^{\prime}\right)
$$

On reprend le calcul au niveau du cas 2 :

$$
\begin{aligned}
\mathcal{R}_{p} \mathfrak{A} R^{\prime} V_{2} \pi \mathfrak{T}_{i} 1 \pi & \Rightarrow \mathcal{R}_{p} \mathfrak{A} R^{\prime} V_{3}^{\prime} V_{2} \mathfrak{T}_{i} 1 \pi \\
& \Rightarrow \mathcal{R}_{p} \mathcal{A} \beta 3 \mathcal{R} r \underbrace{\mathcal{H} 3 D \mathcal{A} V_{3}^{\prime}}_{V_{3}^{\prime \prime}} V_{3}^{\prime} \mathfrak{T}_{i} 1 \pi \\
& \Rightarrow \mathcal{R}_{p} \mathcal{A} \underbrace{\beta 3 \mathcal{R} V_{3}^{\prime \prime} I V_{3}^{\prime \prime} V_{3}^{\prime} \mathfrak{T}_{i} 1 \pi}_{i^{\prime \prime}}
\end{aligned}
$$

Si $i^{\prime \prime} \equiv \lambda x(x 0)$ on exécute le cas 2 , sinon on exécute de nouveau le cas 3 . 


\section{VI.2. Opérateurs $\in M$ (modificateurs sur $E$.)}

On se limitera à des opérateurs de retrait et d'ajout agissant sur des éléments $e_{i}$ extrémités de liste.

Opérateur de retrait

On trouvera deux cas de figures et à retirer. Dans un but de simplification, on suppose que la réserve de cellules libres est infinie ou suffisante pour une suite d'opérations quelconques pour éviter le « repêchage » des cellules (garbage collector).

Proposition 7. La sémantique de l'opérateur de retrait RET dans une liste bidirectionnelle peut s'exprimer par un doublet de combinateurs (RET 1, RET 2) avec RET 1 sur $\pi$ et RET 2 sur $D A \pi$ tels que :

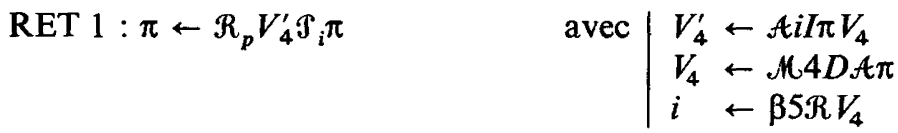

$$
\begin{aligned}
& \text { RET 2:DA } \pi \leftarrow \mathcal{R}_{B} K_{p} \mathfrak{T}_{B} 2 \mathfrak{S}_{m} i^{\prime} D \mathcal{A} \pi \quad \text { avec } \mid \begin{array}{l}
i^{\prime} \leftarrow \beta 2 \mathcal{R} V_{4} I 23 \\
K_{p} \equiv \lambda x(x K)
\end{array}
\end{aligned}
$$

\section{Démonstration :}

$\pi \leftarrow \operatorname{RET~1.~} \pi \quad V_{4}^{\prime}$ est la p.i. $\left(\operatorname{mot}^{4}\right) e_{i}$ donc l'adresse de la cellule $e_{i .1}$

$D \mathcal{A} \pi \leftarrow$ RET 2. DAA RET 2 affecte $c . \mathrm{e}^{2}$. (reliquat $\left.\left(\operatorname{mot}^{i^{\prime}}\right)\right) e_{i}$

$$
\begin{aligned}
& \text { à } \lambda x(x 0) \text { si GAUCHE }\left(e_{i}\right) \neq \varnothing \\
& \text { à } \lambda x(x K) \text { sinon. }
\end{aligned}
$$

$\beta 2 \mathcal{R} V_{4}$ sélectionne c.e ${ }^{2}$. (reliquat $\left(V_{4}\right)$ ).

1) $i^{\prime}=\lambda x(x K) I 23$

$$
\begin{aligned}
& =K 23 \\
& =2
\end{aligned}
$$

2) $i^{\prime}=\lambda x(x 0) I 23$

$$
\begin{aligned}
& =023 \\
& =3
\end{aligned}
$$

\section{Opérateur d'ajout}

A ce niveau, il est indispensable d'introduire l'ensemble « réserve » composé de cellules disponibles liées. Soit $\psi$ le pointeur sur la tête de la réserve.

On observera les deux cas possibles : (les liaisons 1 donnant la situation avant l'opération d'ajout et les liaisons 2 après cette opération). 
Cas $n^{\mathbf{0}} 1$ :

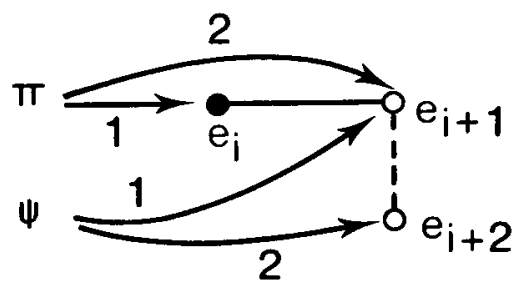

Cas $n^{\circ} 2$ :

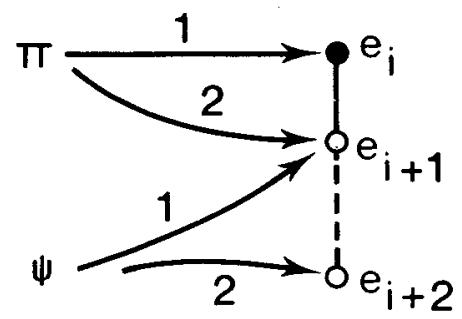

Proposition 8. La sémantique de l'opérateur d'ajout s'exprime par un quadruplet de combinateurs 1-paramétré $(u) a j=\left(a j_{1}, a j_{2}, a j_{3}, a j_{5}\right)$. Ce quadruplet est ordonné :

$$
a j_{1}>a j_{2}>a j_{3}>a j_{4}
$$

Les quatre éléments intervenant dans cette opération d'ajout sont :

$$
e_{i}(D \mathcal{A} \pi), e_{i+1}(D A \psi), \pi, \psi
$$

sur lesquels agissent respectivement : $a j_{1}, a j_{2}, a j_{3}, a j_{4}$.

$$
\begin{aligned}
& a j_{1} \equiv \mathcal{R}_{B} O_{p} \mathfrak{T}_{B} 2 \mathfrak{T}_{m} 2 \mathcal{R}_{p} \mathcal{A} \psi \mathfrak{T}_{i} \mathfrak{T}_{m} u \\
& a j_{2} \equiv \mathcal{R}_{B} K_{p} \mathfrak{T}_{B} 2 \mathfrak{S}_{m} 2 \mathcal{R}_{B} O_{p} \mathfrak{T}_{B} i^{\prime} \mathfrak{T}_{m} 4 \mathcal{R}_{p} \mathcal{A} \pi \mathfrak{T}_{i} \mathfrak{T}_{m} 4 \\
& a j_{3} \equiv \mathcal{R}_{p} \mathcal{A} \psi \mathfrak{T}_{i} \\
& a j_{4} \equiv \mathcal{R}_{p} \mathcal{A} \mathcal{H} 2 D \mathcal{A} \psi \mathfrak{T}_{i}
\end{aligned}
$$

avec :

$$
\begin{aligned}
& O_{p}=\lambda x(x 0) \\
& i^{\prime} \leftarrow \beta 2 \mathcal{R} V_{3} I 25 \\
& V_{3} \leftarrow \mathcal{H} D A A \pi
\end{aligned}
$$

Seul le combinateur $a j_{1}$ est 1-paramétré.

Pour le cas $\mathrm{n}^{\circ} 1, u=2$, pour le cas $\mathrm{n}^{\circ} 2, u=3$. 


\section{Démonstration :}

$a j_{1}:$ 1. Mise en place dans $e_{i}$ de l'adresse du successeur $e_{i+1}$.

2. Indication que $s\left(e_{i}\right) \neq \varnothing$ ou DROITE $\left(e_{i}\right) \neq \varnothing$.

$$
\begin{aligned}
& \mathcal{R}_{p} \underbrace{\mathcal{A} \psi} \underbrace{\mathfrak{T}_{i}^{\mathfrak{T}_{m}} 2 D \AA \pi} \\
& \text { 1.2. } 1.1 \text {. }
\end{aligned}
$$

1.1. Positionnement sur p..$^{2}$. de l'élément dont l'adresse est dans $\pi$, c'est-à-dire $e_{i}$.

1.2. Sélection de p.i. $(\psi)$

1.1. et 1.2. : Affectation de p.i ${ }^{2} .\left(e_{i}\right)$ par p.i. $(\psi)$.

2 :

$$
\underbrace{\mathcal{R}_{B} O_{p}}_{2.2 .} \underbrace{\mathfrak{S}_{B} 2 \mathfrak{T}_{m} 3 D \mathcal{A} \pi}_{2.1 .}
$$

2.1. Positionnement sur la c.e $e^{2}$ (reliquat $\left.\left(\operatorname{mot}^{3}\right)\right) e_{i}$

2.2. Affectation de cette c.e. par $O_{p}=\lambda x(x 0)$.

$a j_{2}:$ 1. Mise en place dans $e_{i+1}$ de l'adresse de $p\left(e_{i}\right)$ ou de GAUCHE $\left(e_{i}\right)$.

2. Indication que $s\left(e_{i}\right) \neq \varnothing$ ou GAUCHE $\left(e_{i}\right) \neq \varnothing$.

3. Indication que $s\left(e_{i+1}\right)=\varnothing$.

Expression de l'action $1: \mathscr{R}_{p} \mathfrak{A} \pi \mathfrak{T}_{i} \mathfrak{J}_{m} 4 D \mathcal{A} \psi$

Expression de l'action $2: \mathfrak{R}_{B} O_{p} \mathfrak{T}_{B} i^{\prime} \mathfrak{T}_{m} 4 D A \mathcal{A}$

$\mathfrak{T}_{m} 4 D \mathcal{A} \psi$ positionne sur $\operatorname{mot}^{4}\left(e_{i+1}\right)$

$i^{\prime} \leftarrow \beta 2 \Re V_{3} I 25$

$V_{3} \leftarrow \mathcal{M} 3 D \mathcal{A} \pi: V_{3} \operatorname{mot}^{3}\left(e_{i}\right)$.

$\beta 2 \Re V_{3}$ : extraction de c. $\mathrm{e}^{2}$. (reliquat $\left(V_{3}\right)$ ).

Deux cas sont possibles :

- Composante : $\lambda x(x 0)$ positionné par $a j_{1}$ cela implique : $a j_{1}>a j_{2}$.

- Composante : $\lambda x(x K)\left(\operatorname{DROITE}\left(e_{i}\right)=\varnothing\right)$

cas $1: \lambda x(x K) I 25 \Rightarrow 2$

cas $2: \lambda x(x 0) I 25 \Rightarrow 5$.

Cette sélection permet de se positionner sur la c.e. correspondante. $\mathrm{n}^{\circ}$ décembre 1974, R-3. 


$$
\begin{aligned}
& a j_{3}: \text { p.i. }(\pi) \leftarrow \text { p.i. }(\psi) \\
& \pi \leftarrow \mathcal{R}_{p} \mathfrak{A} \psi \mathfrak{T}_{i} \pi \\
& a j_{4}: \quad \text { p.i. }(\psi) \leftarrow \text { adresse de } s\left(e_{i+1}\right) \\
& \psi \leftarrow \mathcal{R}_{p} \mathcal{A} \cdot \mathcal{M} 2 D \mathcal{A} \psi \mathfrak{S}_{i} \psi \\
& 21 \\
& 1 \text { : positionnement sur p.i. de } \psi \\
& 2 \text { : extraction dans } e_{i+1} \text { de l'adresse de } e_{i+2} \text {. }
\end{aligned}
$$

Dans le même esprit, il est possible d'exprimer d'autres opérateurs d'ajout et de retrait.plus complexes (cas d'éléments qui ne sont pas des queues de listes).

\section{BIBLIOGRAPHIE}

[1] R. CASTANET, Une formalisation de la sémantique des opérateurs d'extraction dans une arborescence, C. R. Acad. Sc. Paris, série A, 275 (1972), 135-137.

[1A] R. CASTANET, Sur la sémantique des opérateurs d'insertion dans une arborescence, C. R. Acad. Sc. Paris, série A, 275 (1972), 209-212.

[2] A. CHURCH, The Calculi of lambda conversion, Princeton University Press (Princeton), 1941.

[3] H. B. CurRY et R. Feys, Combinatory logic, 1, North Holland (Amsterdam), 1958.

[4] L. Nourn, Logique combinatoire et algorithmes, C. R. Acad. Sc. Paris, série A, 272 (1972), $1435-1438$ et $1485-1488$.

[5] E. V. PADUCEVA, Réduction de groupes de coordination contenant des éléments se répétant, in La sémantique en U.R.S.S., Dunod, Paris (1971).

[6] B. RoBinet, Sémantique des tableaux : Application au langage APL. Thèse $3^{\mathrm{e}}$ cycle, Université Paris, 6 (1972).

[7] R. H. Lethwell and J. E. Mezei, A formal description of APL, Actes du congrès APL, IRIA, Rocquencourt (1971).

[8] M. Weizenbaum, Symetric List Processor, Comm. ACM, vol. 6, $\mathrm{n}^{\circ} 9$ (1963), 524-544.

[9] J. Mac CaRthy, LISP 1.5 Programmer's Manual, MIT Press, Cambridge Mass. (1962).

[10] K. C. Knowlton, A programmer's description of L6. Comm. ACM, vol. 7 (1965), 623-625.

[11] C. Böнм, The CUCH as a formal and description language, in Formal language, description language, STEEL (1964).

[12] P. WeGNeR, Programming, information structures and machine organisation, McGraw Hill, New York (1968).

[13] M. Venturini-Zilli, Lambda $K$ formulae for vectors operators, Int. Comp. Center Bull., 4 (1965), 157-174. 


\section{ANNEXE 1 \\ PRESENTATION DU LAMBDA- $K$-CALCULUS}

\section{Morphologie}

- Les éléments de base sont les variables. Soit $V$ le jeu de toutes les variables. Les autres symboles sont $: \lambda,($,$) . Ces symboles primitifs per-$ mettent de former d'autres symboles par concaténation.

- Les instructions ou mots bien formés du système formel seront appelés lambda expressions.

Les règles qui permettent de définir une $\lambda$ expression sont les suivantes :

- Une variable est une $\lambda$ expression.

- Si $M$ est une $\lambda$ expression et $x$ est une variable, $\lambda x M$ est une $\lambda$ expression.

- Si $A$ et $B$ sont des $\lambda$ expressions $(A B)$ est une $\lambda$ expression où $A$ est la partie opérateur et $B$ la partie opérande.

On définit trois classes de variables :

- Classe des variables de liaison qui suivent le caractère $\lambda$.

- Classe des variables liées, $x$ est lié dans $X$ si et seulement si $x$ est l'argument lié dans $X$ ou une composante de $X$.

- Classe des variables libres, $x$ est libre dans $X$ si $x$ n'apparaît pas comme liée dans $X$.

REMARQUE : La morphologie du $\lambda$ - $K$-Calculus accepte comme bien formée des expressions de la forme : $\lambda x M$ où $x$ n'apparaît pas dans $M$.

\section{Théorie propre du Lambda-K-Calculus}

On se fixe deux règles qui permettent de déduire d'axiomes des théorèmes qui seront des $\lambda$ expressions bien formées.

$\mathrm{n}^{\circ}$ décembre 1974, R-3. 
- Règle de substitution ou de réduction

Les expressions du type $(A B)$ spécifient l'application de l'opérateur $A$ sur l'opérande $B$.

Si $A$ est de la forme $\lambda x M$ cette application est la substitution de toutes les occurrences de la variable libre $x$ dans $M$ par l'opérande $B$.

- Règle de réappélation

Si $M$ est une $\lambda$ expression et si $x$ est une variable liée dans $M$, on peut lui substituer la variable $y$ si $M$ ne contient pas d'occurrences libres de $x$ et si $y$ n'apparaît pas dans $M$. 